Ann. Zootech., I967, 16 (4), 335-34r.

\title{
NOTE RELATIVE A LA COMPARAISON DES DESCENDANCES DE TAUREAUX DANS LE CAS D'ÉPREUVES DISCONTINUES
}

\author{
B. VISSAC \\ Station centrale de Génétique animale, \\ Centre national de Recherches zootechniques, 78 -Jouy-en-Josas \\ Institut national de la Recherche agronomique
}

I. $-O B J F I^{\circ}$

Les épreuves de descendance des taureaux d'insémination artificielle sont généralement réalisées par les centres d'une façon discontinute dans le temps et dans l'espace. Chaque centre met en comparaison périodiquement ses propres taureaux, pour une race donnée : il constitue ce que nous appellerons une "série de testage ". L e schéma expérimental est conçu de façon à concentrer les naissances des produits de chaque taureau d'une série clans une période de temps limitée et dans une région ou dans des fermes choisies comme caractéristiques d'un type de production et de méthodes d'élevage définies. I)ans ces limites, l'échantillon de descendants de chaque taureau est considéré comme tiré au hasard de la population globale.

Ieux méthodes peuvent être utilisées pour comparer les taureaux d'après la valeur moyenne de leurs descendants. IJans la première, on dispose, outre les descendants des taureaux, de leurs contemporains (nés à la même époque dans le même troupeau) : on peut alors exprimer dans chaque série la valeur des produits en écart à la valeur moyenne des contemporains. Cette méthode est justifiée si on suppose que les inséminations sont faites de telle façon que les contemporains constituent un échantillon représentatif de la moyenne de la population. I)ans l'hypothèse où le progrès génétique est faible et où les différences génétiques sont réduites d'une région à l'autre, ce qui est généralement le cas, on peut estimer que cet échantillon de contemporains permet de mesurer les variations dues au milieu clans le temps et dans l'espace et de comparer entre elles les estimées de valeur génétique des taureaux de différentes séries. Cette méthode est précisément appliquée d'une façon générale en production laitière, et notamment en France (PoI,y et al, I965). En production de viande, qu'il s'agisse de contrôle de descendance réalisé en ferme (cas du testage pour la produc- 
tion de veaux de boucherie. VISSAC, Ig64 (I)) ou en station, on ne dispose généralement pas de "contemporains" autres que ceux issus des taureaux de la "série de testage " correspondante. Il est, de ce fait, difficile de comparer la valeur génétique de taureaux appartenant à des séries de testage différentes.

L'objet de la présente note est de déterminer l'efficacité statistique respective de 2 procédés utilisables dans ce but et utilisés effectivement par les centres d'insémination français.

a) Procédé global : on suppose qu'il n'y a pas de différence entre la valeur génétique moyenne des taureaux de deux séries de testage et on compare entre elles les performances moyennes des descendants des mâles de 2 séries, exprimées chacune en déviation par rapport à la performance moyenne des descendants des autres taureaux de leur série.

b) Procédé partiel : on utilise un " taureau témoin " dont les descendants sont contrôlés à 1'occasion de chaque série de testage; les performances moyennes des descendants des taureaux de chaque série sont alors exprimées en déviation par rapport à la valeur moyenne des descendants du taureau témoin pour la série correspondante.

\section{II. -.- PRINCIPE, DE, LA COMPARAISON}

Si on néglige les influences autres que celles de la série de testage et du taureau, le modèle mathématique en cause est le suivant :

$$
\mathrm{Y}_{i j k}=\mu+a_{i}+b_{i j}+c_{i j k}
$$

$\mathrm{Y}_{i j k}=$ performance contrôlée du $k^{\mathrm{eme}}$ veau issu du taureau $j$ dans la série $i$,

$\mu=$ effet moyen général,

$a_{i}=$ variable aléatoire caractérisant l'effet moyen de la $i$ eme série avec :

$$
\mathrm{E}\left(a_{i}\right)=\mathrm{o} \quad \mathrm{E}\left(a_{i}^{2}\right)=\sigma_{a}^{2}
$$

$b_{i j}=$ variable aléatoire caractérisant l'effet moyen du jeme taureau que l'on cherche à caractériser avec : $\mathrm{E}_{\mathbf{l}}\left(b_{i j}\right)=0 \mathrm{E}\left(b_{i j}^{2}\right)=\sigma_{b}^{2}$,

$c_{i j k}=$ variable aléatoire caractérisant la déviation du $k^{\mathrm{em}}$ veau par rapport à la moyenne des veaux issus du $j$ eme taureau dans la $i$ eme série:

$$
\begin{aligned}
& \mathrm{E}\left(c_{i j k}\right)=0 \\
& \mathrm{E}\left(c_{i j k}^{2}\right)=\sigma_{c}^{2}
\end{aligned}
$$

\section{I. - Comparaison de 2 taureaux de la mêtme série:}

Soient 2 taureaux indicés $j:$ I,2 dans la série $i$; les différences entre les estimées de valeur moyenne de leurs descendants sont:

$$
\begin{aligned}
\overline{\mathrm{Y}}_{i 1} \cdot \overline{\mathrm{Y}}_{i 2 \cdot}=\left(b_{i 1}-b_{i 2}\right) & +\sum_{k} \frac{c_{i 1 k}}{n}-\sum_{k} \frac{c_{i 2 k}}{n} \\
= & b_{i 1}-b_{i 2}+e_{1}
\end{aligned}
$$

avec $n$, effectif de descendants par taureau, supposé constant. 
La variance de l'erreur: $\sigma^{2} e_{1}=\sigma_{c}^{2}\left(\frac{2}{n}\right)$

II. 2. - Comparaison de 2 taureaux de séries différentes:

II. I. I. - Procédé partiel (taureau témoin)

soient : $j=0$ l'indice du taureau témoin,

$j=\mathrm{I}$ celui d'un taureau de la série $i=\mathrm{I}$,

$j=2$ celui d'un taureau de la série $i=2$.

( $n, 1$ 'effectif, supposé constant, de descendants pour $j=0, \mathrm{I}, 2$ ).

I a comparaison ci-dessus s'exprime :

$$
\begin{aligned}
&\left(\overline{\mathrm{Y}}_{11} \cdot-\overline{\mathrm{Y}}_{10}\right)-\left(\overline{\mathrm{Y}}_{22} \cdot-\overline{\mathrm{Y}}_{20}\right)=\left(b_{11}-b_{22}\right) \\
& \quad+\sum_{k} \frac{c_{11 k}}{n}-\sum_{k} \frac{c_{10 k}}{n}-\sum^{c_{22 k}}+\sum_{k} \frac{c_{20 k}}{n} \\
&=\left(b_{11}-b_{22}\right)+e_{2}
\end{aligned}
$$

I, a variance de l'erreur devient dans ce cas :

$$
\sigma_{\epsilon_{2}}^{2}=\sigma_{e}^{2}\left(\frac{4}{n}\right) \text { elle est double de celle correspondant à } e_{1} \text {. }
$$

II. I. 2. - Procédé global

La comparaison définie s'exprime :

$$
\begin{aligned}
\left(\overline{\mathrm{Y}}_{11}-\overline{\mathrm{Y}}_{1 . .}\right)-\left(\overline{\mathrm{Y}}_{22}-\overline{\mathrm{Y}}_{2 .}\right)=\left(b_{11}-b_{22}\right)+\left(\sum_{j}-b_{2 j}-\sum_{j} \frac{b_{1 j}}{k_{1}}\right) & \\
& +\left(\sum_{k} \frac{c_{11 k}}{n}-\sum_{k} \frac{c_{22 k}}{n}\right)+\left(\sum_{j k} \frac{c_{2 j k}}{\mathrm{~N}_{2}}-\sum_{j k} \frac{c_{1 j k}}{\mathrm{~N}_{1}}\right) \\
= & \left(b_{11}-b_{22}\right)+e_{3}
\end{aligned}
$$

avec : $N_{1}, N_{2}$, effectifs totaux de veaux contemporains contrôlés dans les séries $I$ et 2 sur tous les taureaux, autres que le taureau concerné; $k_{1}, k_{2}:$ nombre de taureaux contemporains ;

$$
\sigma_{e_{3}}^{2}=\sigma_{b}^{2}\left(\frac{\mathrm{I}}{k_{1}}+\frac{\mathrm{I}}{k_{2}}\right)+\sigma_{c}^{2}\left(\frac{2}{n}+\frac{\mathrm{I}}{\mathrm{N}_{1}}+\frac{\mathrm{I}}{\mathrm{N}_{2}}\right)
$$

Le rapport des variances des erreurs effectuées par chacun de ces 2 procédés est :

$$
\frac{\sigma_{e_{2}^{2}}^{2}}{\sigma_{e 3}^{2}}=\frac{\sigma_{c}^{2}(4 / n)}{\sigma_{c}^{2}\left(\frac{2}{n}+\frac{\mathrm{I}}{\mathrm{N}_{1}}+\frac{\mathrm{I}}{\mathrm{N}_{2}}\right)+\sigma_{b}^{2}\left(\frac{\mathrm{I}}{k_{1}}+\frac{\mathrm{I}}{h_{2}}\right)}
$$

en posant $\frac{\mathrm{I}}{\mathrm{N}}=\frac{\mathrm{I}}{2}\left(\frac{\mathrm{I}}{\mathrm{N}_{1}}+\frac{\mathrm{I}}{\mathrm{N}_{2}}\right)$

$$
\begin{aligned}
\frac{\mathrm{I}}{k} & =\frac{\mathrm{I}}{2}\left(\frac{\mathrm{I}}{k_{1}}+\frac{\mathrm{I}}{k_{2}}\right) \\
h^{2} & =\frac{4 \sigma_{b}^{2}}{\sigma_{b}^{2}+\sigma_{c}^{2}} \text { (coefficient d'héritabilité du caractère considéré). }
\end{aligned}
$$

Annales de Zootechnie. - I 967 . 
on obtient :

$$
=\frac{\frac{\sigma_{e 2}^{2}}{\sigma_{e 3}^{2}}=\frac{\sigma_{c}^{2}\left(\frac{2}{n}\right)}{\sigma_{c}^{2}\left(\frac{\mathrm{I}}{n}+\frac{\mathrm{I}}{\mathrm{N}}\right)+\sigma_{b}^{2}\left(\frac{\mathrm{I}}{k}\right)} \text { avec } \frac{\mathrm{I}}{\mathrm{N}}=\frac{\mathrm{I}}{n k}}{\left(\frac{\mathrm{I}}{k}+\mathrm{I}\right)+\frac{n}{k}\left(\frac{h^{2}}{4-h^{2}}\right)}
$$

\section{III. - APPLICATION}

L'intérêt respectif des 2 procédés étudiés dépend donc, sous les hypothèses for-. mulées, des 3 paramètres : $n$, $k$ et $h^{2}$ c'est-à-dire :

- de l'effectif des descendants par taureau,

- de l'effectif des taureaux de chaque série,

- du coefficient d'héritabilité.

TABLEAU I

$V$ aleurs de $\sigma_{e 2}^{2} \sigma_{e \mathrm{a}}$

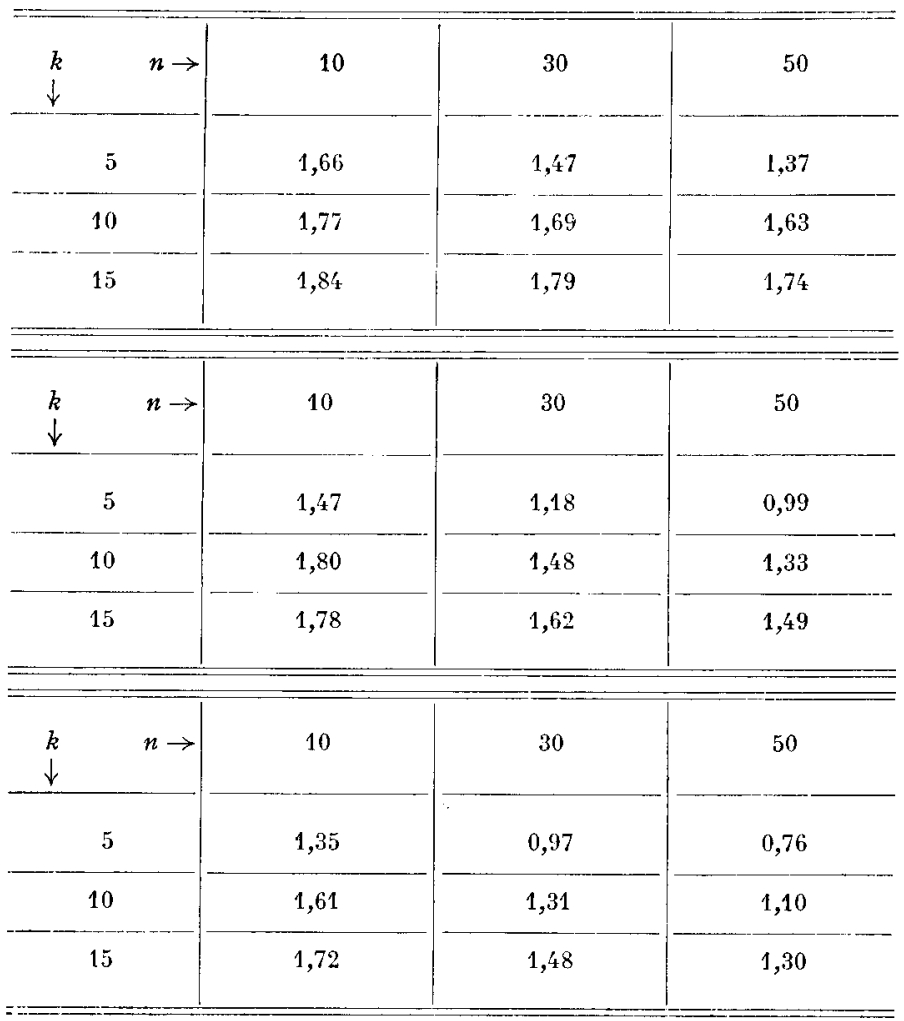


Plus l'effectif par taureau et le coefficient d'héritabilité sont élevés, plus la méthode du taureau témoin est intéressante, par contre plus les séries de taureaux mis en comparaison sont importantes plus la méthode globale est préférable.

Nous déterminerons successivement, pour des combinaisons de valeurs des 3 paramètres ci-dessus les plus fréquentes en pratique $(n=\mathrm{I} 0,30,50 ; k=5$, I0, I5 ; $h^{2}=0$, ro, $\left.0.30,0.50\right)$ :

Io les valeurs du rapport : $\sigma_{e 2}^{2} / \sigma_{e 3}^{2}$

$2^{\circ}$ le rapport des effectifs qu'il faudrait contrôler pour le taureau témoin $\left(n^{\prime}\right)$ par rapport à l'effectif contrôlé par taureau $(n)$ pour obtenir la même précision par les 2 méthodes $\left(\sigma_{e 2}^{2}=\sigma_{\ell 3}^{2}\right)$

\section{TABLEAU 2}

Rapport des effectifs à contrôler pour le taureau témoin $\left(n^{\prime}\right)$ et les autres taureaux $(n)=\frac{n^{\prime}}{n}$

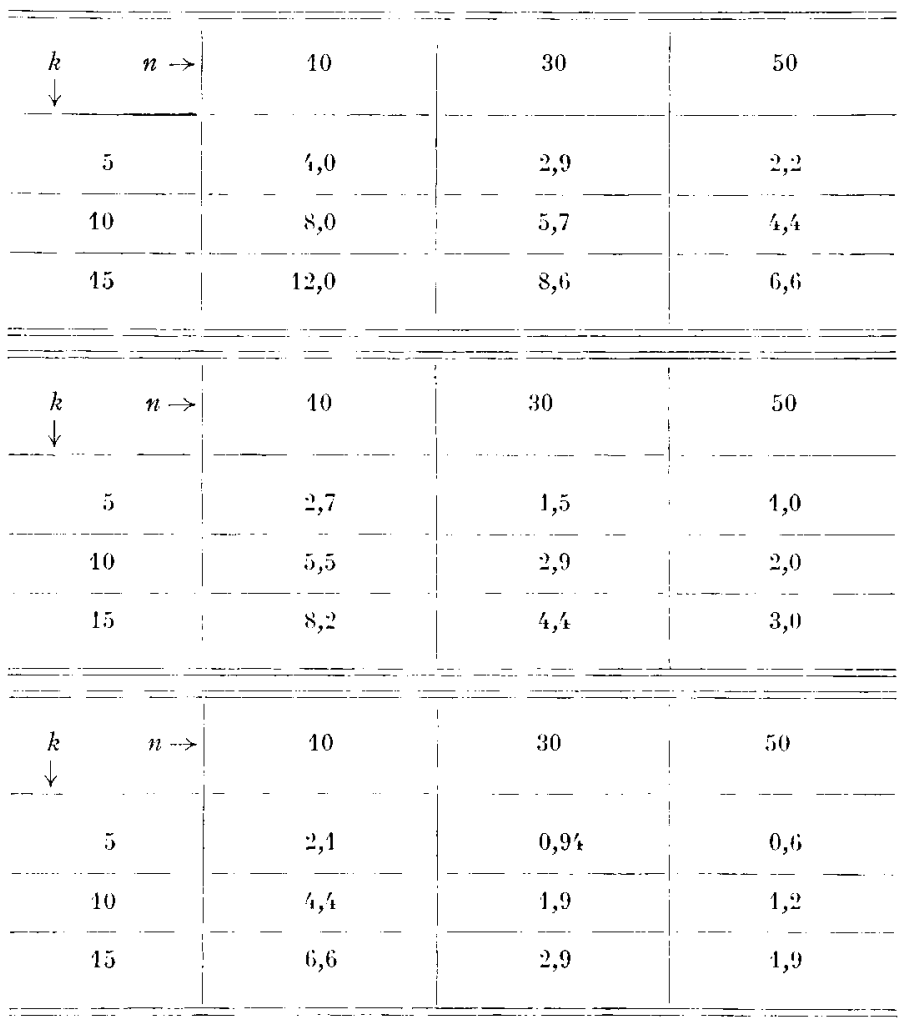

III. I. - Valeurs $d u$ rapport $\sigma_{e 2}^{2} / \sigma_{e 3}^{2}$

I.es valeurs obtenues figurent sur le tableau $\mathrm{r}$.

Compte tenu des limites de variation de $h^{2}$ constatées en pratique, il ressort de res résultats que :

-- dans le cas d' $h^{2}$ faibles $(0,10)$ la comparaison à l'ensemble des taureaux contemporains est toujours largement préférable à celle du "taureau témoin "; 
- dans le cas d' $h^{2}$ moyennes $(0,30)$, c'est également le cas lorsque $n<50$ et $k>5$ c'est-à-dire dans le cas de séries de testage portant sur un nombre élevé de taureaux et un effectif de descendants faible par taureau;

-- lorsque le coefficient d'héritabilité est élevé $\left(h^{2}=0,50\right)$, pour le testage en station par exemple, l'intérêt du taureau témoin est beaucoup plus évident : cette méthode est préférable pour $k<8$ et $n>25$.

\section{2. - Rapport des effectifs à contrôler pour le taureau témoin et les autres taureaux.}

L'égalité du rapport des variances est obtenue, en supposant un effectif de descendants différent pour le "taureau témoin " $\left(n^{\prime}\right)$ par rapport aux taureaux mis au testage $(n)$, avec $n^{\prime}==\frac{k}{\mathrm{I}-\frac{h}{n}+\frac{h^{2}}{4-h^{2}}}$.

Les valeurs du rapport $n^{\prime} / n$ pour les diverses combinaisons de $k, n$ et $h^{2}$ figurent sur le tableau 2 .

\section{IV. - CONCLUSION}

Lorsque le contrôle de descendance des taureaux d'insémination porte sur des séries discontinues dans le temps et dans l'espace, et lorsqu'on ne dispose pas de produits contemporains dans la même ferme, la comparaison des mâles à la moyenne de la série contemporaine est, en général et théoriquement, préférable à la comparaison de ces derniers à un " taureau témoin ".

Toutefois ce résultat théorique n'est valable que si les différences génétiques moyennes entre taureaux de plusieurs séries sont analogues à celles qui apparaissent entre taureaux intra-série. Cette hypothèse suppose en particulier qu'il n'y a pas de progrès génétique dans le temps et que les méthodes de choix des mâles utilisées par chaque centre sont soit équivalentes, soit sans effet sur les caractères considérés.

L'emploi d'un " échantillon témoin " concuremment à la méthode globale se justifierait alors, pour tenir compte des différences génétiques entre séries, que nous ne pouvons apprécier. Il serait dans ce but aussi valable de considérer comme "échantillon témoin "non la descendance d'un seul taureau, mais celles de taureaux déjà testés par les différents centres, et dont une certaine quantité de sperme serait stockée pour être utilisée plusieurs années par tous les centres pratiquant le testage. Ians le cas d'un testage pratiqué sur 50 veaux par taureau, l'effectif de veaux de cet échantillon témoin devrait être pour avoir par cette méthode une précision au moins équivalente à la méthode globale, de I à 5 fois plus élevé suivant l'effectif de taureaux mis en comparaison et la valeur du coefficient d'héritabilité. 


\section{SUMMARY}

TWO METHODS FOR COMPARING THE PROGENIES OF DISCONTINUOUSLY TESTED BULLS

The accuracy of two methods for comparing the progenies of Bulls tested in discontinuous series in time or space was studied when no information was available on the performances of the contemporaneous animals tested in the same farms (this is the French case of progeny-testing of beef-bred bulls for the value of their calves).

With the Ist method 'The average performances of the progenies of 2 bulls belonging to 2 different series are expressed in relation to a progeny-tested control bull of each series. With the and method, the average performances are expressed in relation to that of all other bulls of the same series.

The accuracies of the two methods are expressed and compared depending upon the number of bulls in each series of test, the number of offspring per bull, and the coefficient of heritability of the character considered.

Under the most common practical conditions of testing beef-bred bulls for the value of their calves (more than 5 bulls per series, 50 calves per bull, coefficient of heritability ranging from o.ro to 0.30 ) the and method appears better in some respects.

\section{RÉFÉRENCES BIBLIOGRAPHIQUES}

Poly J., Poutous M., Frebling J., 1965. Méthodes de calcul d'index de production laitière. Bull. tech. Inf. Ingrs. Sens. agric., 205, 957-963.

VIssac B., I964. Méthode de détermination de l'index génotypique des taureaux d'insémination sur la valcur de leurs veaux de boucherie. Ann. Zootech., 13, 267-275. 